Results A total of 50 patients were studied (mean age 62.7 years, SD 13.2). $60 \%$ of patients showed at least one reconciliation error. The average number of drugs involved in reconciliation errors was 1.8 (SD 1.2) per patient. A total of 54 (17\%) drugs discrepancies were found. The most common error was omission of a regularly used medicine (74\%), followed by discrepancies in the frequency (9\%), incorrect drug (9\%) and incorrect dose (8\%). Antihypertensive drugs represented $37 \%$ of all discrepancies. Pharmacists made interventions in $98 \%$ of discrepancies. Most pharmacist interventions consisted of the addition of an omitted drug (66\%) and dosage adjustment (9.4\%). 81\% of recommended interventions were accepted by ICU physicians. Most rejected interventions were due to the patient's clinical status (70\%).

Conclusions Critically ill patients showed a high incidence of medicines reconciliation errors. Most reconciliation errors consisted of omissions of chronic medicines and involved antihypertensive drugs. $81 \%$ of pharmacist interventions were accepted. Medicines reconciliation could reduce medicines errors in critically ill patients and should be incorporated into the daily routine of the pharmacist responsible for the unit.

No conflict of interest.

\section{CPC-130 SWITCHING FROM ADEFOVIR TO TENOFOVIR IN HEPATITIS B-INFECTED PATIENTS}

doi:10.1136/ejhpharm-2013-000276.587

B López García, E Fernandez Colominas, R Pellicer Voltas, S Ortonobes Roig, 0 Ferrandez Quirantes, A Carmona Yelo. Hospital del Mar, Pharmacy, Barcelona, Spain

Background Adefovir disoproxil (ADF) was the second nucleoside analogue to be approved for Hepatitis B Virus (HBV) treatment. Later studies showed that tenofovir had better and more cost effective clinical outcomes for HBV treatment.

Purpose To analyse the treatment changes in patients with chronic infection on $\mathrm{ADF}$ treatment. To define treatment changes and their clinical causes and effects in our population.

Materials and Methods A retrospective observational study was performed in a tertiary hospital including all patients treated with ADF between January 2005 and September 2012. Data collected: demographics (sex, age) previous treatment, ADF treatment duration, reasons for changing from $\mathrm{ADF}$, new drug prescribed, $\mathrm{HBV}$ DNA viral load at the moment of change and 6 months later.

Results Fifty-nine patients started treatment with ADF during the study period; men (81.4\%), mean age: 42 years. Previous treatment: 45 treatment-naïve, 2 Peginterferon-a2-a and 12 lamivudine. Fourteen patients were lost to follow-up. Of the 45 patients included mean duration of treatment with ADF was 44.96 months (range: 3-92). 40 patients changed treatment with ADF: 27 patients switched to TDF with undetectable HBV DNA viral load (two of them returned to $\mathrm{ADF}$ due to intolerance); 15 patients switched due to detectable HBV DNA-viral load: 10 patients to TDF and 5 to ETV. 5 patients remain on $\mathrm{ADF}$.

Conclusions Nearly every patient treated with ADF has changed treatment at some point and are no longer treated with this drug.

Most patients switched from ADV to TDF without any clinical reason; this may be related to better clinical outcomes and cost effectiveness.

No conflict of interest.

\section{CPC-131 SWITCHING STRATEGY. THE PHARMACIST'S POINT OF VIEW ON COST, ADHERENCE AND VIROLOGICAL OUTCOME}

doi:10.1136/ejhpharm-2013-000276.588

${ }^{1} \mathrm{C}$ Carcieri, ' $\mathrm{G}$ Cinnirella, ${ }^{1} \mathrm{~S}$ Bertini, ${ }^{2 B}$ Salassa, ${ }^{2} \mathrm{M}$ Bonasso, ${ }^{2} \mathrm{C}$ Bramato, ${ }^{2} \mathrm{G}$ Orofino, ${ }^{2} \mathrm{~S}$ Carosella, ${ }^{2} \mathrm{G}$ Leo. ${ }^{1}$ Ospedale Amedeo di Savoia, Hospital pharmacy, Turin, Italy; ${ }^{2}$ Ospedale Amedeo di Savoia, Ambulatory of Infectious Disease, Turin, Italy
Background HIV infection is a complex medical problem that requires careful monitoring of adherence to treatment, efficacy, development of resistances and toxicities. The estimated life expectancy of patients is increasing, this makes it necessary to find efficient ways to optimise switching therapy away from HAART in order to reduce costs and increase its efficacy. At Amedeo di Savoia Hospital of Turin, the regional centre in Piedmont for HIV infection diagnosis and treatment, hospital pharmacists work in a multidisciplinary team with infectiologists, nurses, psychologists and dieticians. The team follows every aspect of the clinical pathway, leading to an improvement in the clinical management of HIV patients.

\section{Purpose}

- To monitor HAART for all patients on therapy

- To identify patients with a switch of therapy

- To create a multidisciplinary database including adherence, economic and clinical data before and after the switch

- To monitor the distribution of available resources in relation with virological outcome and adherence response of patients

- To achieve a rational use of resources

Materials and Methods Collecting data from Oliamm Software and File F using a specific software application, we analysed cost and adherence by the pharmacy refill method (days supplies between refill dates/duration of dispensed therapy $x$ 100) for each switch of treatment between March 2010 and March 2012. From clinical reports we also evaluated the reasons for switching (toxicity, simplification, treatment failure) and the success of variation in term of virological outcome.

Results Switching of antiretroviral treatments occured in 250 patients (male 177, female 73, median age 48 years), out of about 1,835 HIV-positive people in treatment, considering overall 310 switches (about $8 \%$ at the patient's request). In 151 cases the switch led to a financial saving and in 159 cases to an increase in cost, leading overall to an excess of cost of $4396,6 €$ each month (an additional $€ 17,59$ for each patient for each month). The reasons for the variation were: treatment failure in $30 \%$, simplification of the treatment in $20 \%$, toxicity in $44 \%$ and other causes in $6 \%$. Focus on simplification evidenced: $13 \%$ decrease in pill burden, $17 \%$ on STR, $55 \%$ on LDR, $10 \%$ on $\mathrm{OD}$ therapy. We also analysed the causes of toxicities. From our study we observed an increased number of patients with suppressed viral load (from $60 \%$ to $77 \%$ ) as evidence of efficacy. 67 out of 125 patients (54\%) with pre-switch viral load non-suppressed, had a suppression after switch. 172 patients out of 185 patients (93\%) with pre switch viral load suppressed, conserved suppression after switch, but 13 patients (7\%) had viral rebound. The change of the treatment didn't impact on adherence in $50.32 \%$ cases and produced an improvement in adherence in $39.03 \%$ of switched patients. Only in $10.65 \%$ did a decrease of adherence improve. We have also analysed the cost distribution, observing a better use of resources to obtain a viral load under $20 \mathrm{cp} / \mathrm{ml}$ and a financial saving for treatment of patients already suppressed pre-switch.

Conclusions In our study an analysis of switching treatment has demonstrated a correct distribution of budget and an improvement in adherence. It has also demonstrated the importance of working in team for better management of the therapeutic path.

No conflict of interest.

\section{CPC-132 SYNDROME OF INAPPROPRIATE ANTIDIURETIC HORMONE SECRETION AS ADVERSE DRUG REACTION IN HOSPITALISED PATIENTS TREATED WITH TOLVAPTAN}

doi:10.1136/ejhpharm-2013-000276.589

P Suarez Artime, C Crespo-Diz, E Espino-Paisan, H Esteban-Cartelle, J Gonzalez Lopez, T Rodríguez-Jato. Complejo Hospitalario Universitario de Santiago de Compostela, Pharmacy, Santiago de Compostela, Spain

Background Some drugs can cause Syndrome of Inappropriate Antidiuretic Hormone Secretion (SIADH). Tolvaptan is a new drug 